\title{
A Biosymtic (Biosymbiotic Robotic) Approach to Human Development and Evolution
}

\author{
Marta Ferraz \\ Faculdade de Ciências e Tecnologia/Nova Universidade de Lisboa, Media Ground, \\ Almada, Portugal \\ atomicdesigners@gmail.com
}

\begin{abstract}
We demonstrate that the current Child-Computer Interaction paradigm is not potentiating human development to its fullest. It is linked to several physical and mental health problems and appears not to be maximizing children's cognitive development and performance. To potentiate children's physical and mental health (including cognitive development and performance) we conceived a new approach to human development and evolution. This approach proposes a particular synergy between the developing human body, computing machines and natural environments. It emphasizes that children should be encouraged to interact with challenging physical environments offering multiple possibilities for sensory stimulation and increasing physical and mental stress to the organism. We created and tested a new set of robotic devices to operationalize our approach - Biosymtic (Biosymbiotic Robotic) devices.
\end{abstract}

Keywords: Child-Computer Interaction - Human development and evolution . Physical and mental health $\cdot$ Cognition $\cdot$ Biosymtic $\cdot$ Natural environments

\section{Introduction}

Hominids evolved while moving in order to survive. Hunter-gatherer children were very active, constantly interacting with challenging natural environments - information processing occurred in a multisensory world. They were nomadic, had to elude predators, walked and ran long distances to forage [25]. Conversely, modern children spend most of their time in artificially controlled environments (ACE; indoors), interacting with screen-based computer devices (SBCDs), stimulating mostly the visual and auditory senses and instilling sedentary behavior $[27,36]$. The Digital Age was accompanied by a strong decline in physical activity (PA) in modern children. This decline sparked multiple physical and mental health issues [25]. Furthermore, it seems that whole-body interaction [30] in natural environments [13] still benefits children's cognitive function (e.g., conceptual knowledge, attention and memory functions) compared to sedentary interaction indoors.

The following section describes the physical and mental health issues linked to the use of SBCDs in ACE and real-world settings (including why cognitive development/performance are not being optimized). Our approach is described in Sect. 2 - a solution based on the employment of Biosymtic devices. Conclusions are then presented in Sect. 3. 


\subsection{Sedentary and Whole-Body Motion Screen-Based Computer Devices in Artificially Controlled Environments and Mobile Screen-Based Computer Devices in Real-World Settings}

Sedentary SBCDs encourage children to experience visual-auditory information-gathering scenarios. Children typically engage SBCDs via user interfaces requiring handeye coordination skills (HECS; e.g., mouse/keyboard; multi-touch). HECS (e.g., tap, drag, slide) allow controlling virtual environments on a two-dimensional (2D) display. Interaction with sedentary SBCDs is linked to improvements in children's cognitive function, e.g., attentional control, selective attention and creativity [18, 19]. However, researchers have shown sedentary SBCDs to be linked with a diversity of physical and mental health complications in children.

Overuse of sedentary SBCDs is associated with obesity, aggressive behaviors, anxiety, increased risk of depression, hyperactivity, attention problems, sleep disorders, attachment and obsessive-compulsive disorders, hand-arm vibration syndrome, bodily discomfort, reduction of physical and emotional awareness, poor fitness, photosensitive epilepsy, visual problems and motion sickness [1, 8, 11,39].

We also suggest that sedentary SBCDs, in ACE, may fail to maximize children's cognitive development and cognitive performance.

Aerobic-based PA and physical exercise (PE), at moderate-intense levels, tend to optimize cognitive function (e.g., attention and memory functions, including academic achievement) and cognitive structure (e.g., increasing hippocampal and basal ganglia volumes - improving cognitive control/response resolution) in children (post-activity benefits), compared to sedentary conditions [7, 21]. Furthermore, while frequently interacting with sedentary SBCDs, in ACE, children get exposed to restricted sensorimotor experiences - restricting the perceptual process (identification, interpretation and organization of sensory information) to auditory and visual events. The child loses the possibility of interacting with a variety of sensory information that natural physical environments (NPE) have to offer - stimulating the visual, auditory, chemical, cutaneous, proprioceptive and vestibular senses [6].

Embodied Cognition research demonstrates that sensorimotor experiences with requirements beyond fine motor skills (demanding small muscular groups and precise actions) tend to improve children's cognitive development/performance. For example, the development of gross motor skills (demanding large muscular groups and less precise actions) improves the development of cognitive function and optimizes academic achievement [34]; gesturing reduces mental effort and facilitates memory retrieval during problem solving [17]. We observed that whole-body interaction in natural environments benefits children's cognitive function (attention and memory functions) compared to sedentary interaction indoors (as we will later describe).

In addition, depriving the child from interaction with multisensory environments may delay or eliminate her ability to integrate multiple sources of sensory information throughout development (difficulty in generating coherent percepts from the physical world and difficulty acting in it) [6, 24].

Whole-body motion (WBM) devices provide enhanced sensorimotor experiences compared to sedentary SBCDs. However, it seems that WBM devices are not promoting 
children's physical and mental health and may fail to maximize their cognitive development/performance.

WBM interfaces make use of whole-body movements in ACE to control virtual information on computer devices, e.g., visual and audio data control can be accomplished through the use of gesture-based and/or sensor-based interfaces [33]. Computer games requiring physical effort are characterized as exergames or active video games (AVGs) and include WBM interfaces [32].

Active Healthy Kids Canada conducted a meta-analysis of the long-term effects of AVGs on children's health and behavior (age 3 to 17 years). This analysis comprised 1367 published papers dated from 2006 to 2012, including 1992 participants from 8 countries. It concluded that AVGs are not promoting the 60 min of moderate to vigorous PA (MVPA) necessary to benefit children's physical and mental health. There was also a lack of evidence suggesting long-term spontaneous adherence to AVGs [23]. Other studies have supported these findings [3].

Because WBM devices, in ACE, are not promoting MVPA levels, they may also be failing to benefit children's cognitive function/structure. WBM devices, in ACE, restrict sensorimotor experiences. While moving, children are encouraged to center attention on a 2D display, depriving them from a variety of motor experiences (proprioceptive and vestibular stimuli) available in three-dimensional space (limiting possibilities to coordinate lower and upper limbs, head and eyes in the physical world). Sensor-based interfaces typically integrate similar materials and textures (e.g., plastic or rubber), limiting tactile experiences. There is also a lack of stimuli variation to the nerve network at the bottom of the feet, as ACE tend to lack textural diversity. Multisensory experiences tend to improve children's cognitive development/performance.

Furthermore, while interacting with SBCDs and WBM devices, in ACE, face-toface and body-to-body interaction (physical contact) appears to be impaired by the continuous focus on a 2D display - weakening the channels of bodily communication. Children who lack human physical contact are more prone to develop anxiety, depression and aggressive behaviors [31].

Children who frequently interact computer devices, in ACE, may also lose benefits from natural ultraviolet radiation (B UVB) - vitamin D for blood level normalization, increased bone mineral density and autoimmune disease prevention. Significant vitamin D deficits may cause bone deformities and fractures [35]. ACE reduce children's contact with germs, diminishing stress on their immune system, which requires contact with germs to properly mature. ACE are associated with a diminished production of antibodies that protect children from life-threatening pathogens [25]. Lack of exposure to natural bright light may also compromise the development of the visual system [37].

SBCDs have been connected to real world settings - Pervasive Computing, e.g., through the use mobile computing devices (MCDs). However, we suggest that these devices may be associated with physical health problems in children and may not be maximizing their cognitive performance. Pervasive Computing unobtrusively connects users and digital worlds [29], e.g., encouraging children to move in the physical space while using MCDs [10, 15].

Prolonged interaction with SBCDs placed lower or higher than the child's gaze is linked to posture issues - burdening upper limbs and neck [12]. MCDs encouraging the 
child to continuously center attention on a 2D display (placed lower than their gaze) may also strain upper limbs and neck. Humans evolved to move in space by maintaining an efficient body posture: neck straight in an upright position [25]. We alert for possible postural problems linked to the use MCDs encouraging movement while focusing on a $2 \mathrm{D}$ display. In addition, by hand holding these devices, children restrict upper limb motor actions (limiting interaction with the physical world).

MCDs typically induce split-attention effect - alternating attention from the device to the physical world. Conversely, external information sources, combined in space and synchronized in time, tend to optimize perceptual integration and improve learning [26, 41]. Hence, MCDs may fail to maximize cognitive performance.

\section{A Biosymtic (Biosymbiotic Robotic) Approach to Human Development and Evolution}

Our approach proposes a particular combination between the human body, computing machines (CMs) and NPE in order to potentiate children's physical and mental health including cognitive development/performance.

\subsection{Potentiating Children's Cognitive Development - Perceptual Abilities - Through Computing Machines}

We suggest two formats of interaction with CMs in order to potentiate the development of perceptual abilities in children:

- Whole-body motion interfaces that allow for multiple possibilities of bodily action in the NPE (the human body as an interface)

Throughout development, children's ability to direct behavior and generate coherent percepts from the environment benefits from multisensory experiences in the physical world - linked to the development of higher-order cognition, e.g., attention, memory and inhibition [6, 24, 42]. Hence, to optimize the development of perceptual abilities, CMs should encourage children to be exposed to multiple bodily action possibilities in multisensory environments. CMs should be combined with WBM interfaces: the child is encouraged to perform a variety of movements (e.g., running, jumping, throwing) to interact with virtual information on CMs, while experiencing the NPE.

- Whole-body motion interfaces that extend the possibilities of bodily action in the NPE (extending physical interfaces)

"Affordances" represent environmental possibilities for action. Perception is a process extended to the structural characteristics of the body and the environment where action evolves [16]. Species perceive the physical world according to their body structures, e.g., a chair suggests "seating" for most humans, a crocodile will probably not notice it $[4,9]$. Changes in the structural features of the human body while maturing, and corresponding action possibilities, influence perception - how children identify, interpret and organize environmental sensory information [42]. We suggest that by 
structurally augmenting the human body, through extending physical interfaces offering new action possibilities (new body structure layout), the child may generate perceptual representations of the environment throughout development, beyond those of the standard human body. We defined this augmentation as "Motoric-Metamorphosis" - a transformation through which the child may generate novel ways of representing and understanding the environment.

CMs should encourage children to experience new action possibilities in enhanced sensory environments (NPE): the child is encouraged to perform new types of bodily action through extending physical interfaces (e.g., moving horizontally in a vertical position, grabbing an object with three arms), which in turn, control virtual information on CMs, while experiencing the NPE.

\subsection{Potentiating Children's Cognitive Performance Through Computing Machines - Combining Computing Devices with Human Biology}

We suggest an adequate combination between $\mathrm{CMs}$ and the human anatomical structure (sensory organs) to avoid postural problems and split-attention effects in children. For example, visual virtual information should be directly aligned with the visual system (e.g., see-through head-mounted displays). The combination between virtual information and the human body should also enable free body movement in the physical world - to freely interact with multiple sources of sensory information (multisensory experiences tend to improve children's cognitive function). We describe this combination as "Virtual-Sensorimotor Alignment" (VSMA).

\subsection{Potentiating Children's Physical and Mental Health Through Computing Machines}

Children should do at least 60 min of daily MVPA to benefit physical and mental health [46]. As previously mentioned, MVPA optimizes children's cognitive function/structure.

A high heart rate (HR) frequency correlates with high levels of PA [2]. HR frequency may vary due to emotional states or excitement levels [38]. Children's HR values increase in situations of excitability or fear: may increase $20-40$ beats per minute (BPM) above the actual resting value [28]. PA in hot or humid climates tends to increase HR values (15 to $20 \mathrm{BPM}$ higher than in neutral climates). HR values also tend to be higher if the produced mechanical work includes gross motor skills [38]. The use of equipment and larger spatial areas increases children's PA levels [44].

Hence, to increase PA levels, children should be encouraged to perform activities in large spatial areas, such as NPE, offering climatic variation (to increase HR values). As the use of equipment may increase PA, children should also be encouraged to interact with a variety of physical tools (user interfaces) fostering the use of gross motor skills. Because children's HR values may also increase in situations of excitability or fear, video game play may be an optimal solution to produce this effect. In fact, children refer to video games as engaging mainly due to the opportunity of experiencing challenging fantasy worlds [20]. 
CMs should promote body-to-body and face-to-face interaction to avoid anxiety, depression and aggressive behaviors and improve bodily communication in children (achieved via VSMA). CMs should also encourage interaction with NPE, as natural bright light improves the development of the visual system. NPE are associated with vitamin D synthesis; stress to the immune system; reduce stress levels and aggressive behaviors and improve children's cognition (restore attention levels) [22].

\subsection{Biosymtic (Biosymbiotic Robotic) Devices}

Our approach is operationalized through a new form of robotic devices, termed Biosymtic (Biosymbiotic Robotic) devices. The central goal of a Biosymtic device (BSD) is to potentiate children's physical and mental health (including cognitive development/performance), while helping them connect with challenging natural environments offering multiple possibilities for sensory stimulation and stressing the multiple systems of the organism [e.g., skeletomuscular, cardiovascular, immune, endocrine and nervous systems (including mental and emotional arousal)].

A BSD is characterized as an artificial system (ranging from physical robots to virtual software agents) displaying automatic control functions while (two modes):

(1) Directly connected to a human organism (human-integrated automatic control);

(2) Disconnected from a human organism (working as an autonomous robot).

In mode 1, a BSD encourages a child to be physically active in NPE through automatic feedback control mechanisms - included in the system's hardware and/or software programs (e.g., video games). A BSD encourages the child to achieve a certain physiological state (e.g., MVPA) in order to improve physical and mental health. Physiological data is always used to persuade a covert response in the child: physical action (an example will be later detailed).

In mode 2, a BSD builds autonomous functions through interaction with a human. We termed this process Bio-Kinesthetic Programming (BKP). BKP is an approach to Robot Programming by Demonstration aiming to help a robot build autonomous functions through human guidance techniques, such as whole-body interaction (e.g., the child's locomotion works as an example to be replicated by the device during autonomous navigation) and physiological states transfer (e.g., the robot learns to manage its power sources according to the child's metabolism), while physically and mentally benefiting the human organism. Hence, a BSD and a human form a symbiosis: the association, or close union, between organisms in mutual benefit [5].

A BSD may also encourage a child to perform PA in a NPE without external control of physiological states - encouraging exploratory play. That is, a child may interact freely with software programs allowing exploratory action in the NPE. These programs allow access to physiological, motion and environmental data, in real-time, e.g., through visual and auditory information (e.g., HR, brain activity; motor performance; humidity, temperature). While accessing a variety of data, the child is encouraged to explore/ regulate her body processes in relation to environmental ones. For example, she may draw inferences about the relations between motion, HR and temperature/humidity in the environment (e.g., HR increasing in humid and hot climates for similar motor 
performance); relations between particular contexts (e.g., mountainous areas; forested areas) and brain states (e.g., alertness, distraction).

We developed and tested two Biosymtic devices to assess the premises of our approach: "Cratus" and "Albert".

The BSD "Cratus" mimics a Roman gladiator/inventor. The physical structure of this device consists of a head connected to a torso, with a wheel mechanism on its base. It also includes a computer processor, on the center back of the torso, that outputs visual and audio information (aligned with the child's visual system - VSMA). System inputs (to control virtual information, e.g., put a video game avatar into action) are made through whole-body motion, e.g., the child may push, pull, rotate and throw the apparatus while running, jumping or trotting. The child may also skate while using this system - feet placed on top of the wheelbase (Motoric-Metamorphosis). The system includes wireless sensors to capture motion data (e.g., accelerometer; moving the system on the terrain translates as virtual locomotion of the game avatar). The system also captures physiological data - e.g., communicates wirelessly with a HR biosensor placed on the child's chest (see Fig. 1).

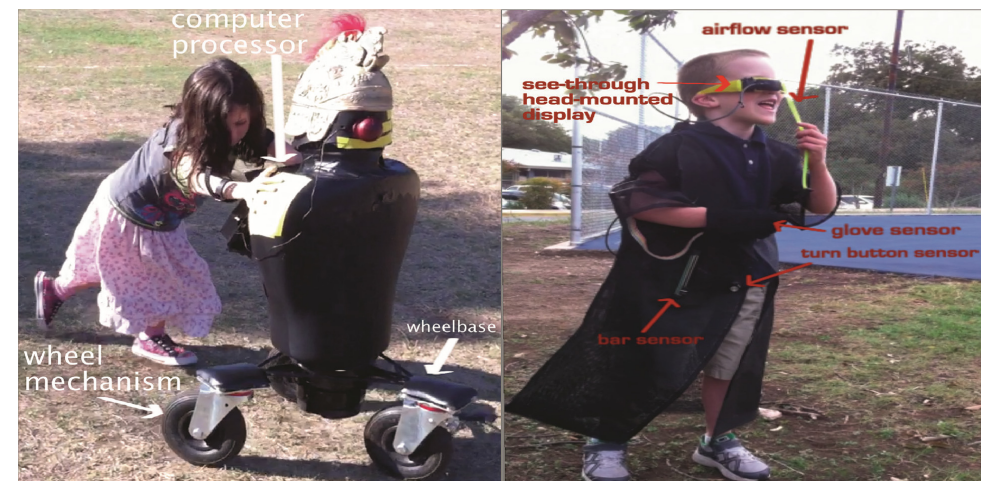

Fig. 1. Left. "Cratus". A child pushes "Cratus" to put a game avatar into motion (visualized on the computer processor); Right. "Albert". A child blowing air into an airflow sensor to put a game avatar into motion (visualized on a see-through head-mounted display).

"Cratus" includes automatic feedback control mechanisms (software) that encourage certain physiological states, e.g., MVPA while playing video games. In the latter, a software actuator controls changes in the displacement speed of the game avatar to encourage MVPA. For instance, if the child engages in low levels of PA (sedentary, measured via HR sensor), while interacting with "Cratus" (e.g., pushing it), the system increases the inertial forces applied to the avatar - the child needs to move faster to reach MVPA. The system also includes a verbal actuator producing audio output. If, for instance, the child engages in low levels of PA, the verbal actuator emits specific feedback, e.g., "Run faster!" or "Give me more power!".

In one cross-sectional study ("Increasing Children's Physical Activity Levels Through Biosymtic Robotic Devices") - presented in [14] - we evaluated the effects of "Cratus" on the PA levels of a group of 20 typically developing children aged 6 to 8 (9 
male and 11 female). Children interacted freely with "Cratus" in a natural forested landscape - playing a video game encouraging MVPA. Children's expectations and opinions regarding the device were also assessed. Results indicated that interacting with "Cratus", in a NPE, instilled vigorous PA levels. Children mentioned they were highly motivated to interact with "Cratus".

The latter results demonstrate that "Cratus", and the NPE, seem a promising solution to promote physical and mental health in children aged 6 to 8 . This study shows that to increase physical activity, children aged 6 to 8 should be encouraged to perform activities in large spatial areas, such as natural environments, and to interact with user interfaces promoting gross motor skills. Because situations of excitability or fear may increase heart rate values, video game play seems an optimal solution to raise physical activity levels. As mentioned, WBM devices in ACE are not promoting MVPA necessary to benefit children's physical and mental health. Similarly, physical education classes and recess programs (spontaneous physical activity), in elementary schools, are not promoting MVPA [40, 43]. "Cratus" may help children increase their daily PA and contribute to a healthy lifestyle. This device could be included in physical education classes (outdoors), recess programs or even help children in hospital environments (e.g., diagnosed with metabolic disorders such as obesity, type 2 diabetes).

The BSD "Albert" consists of a wearable suit with wireless sensors communicating with a computer processor. It includes a monocular see-through head-mounted display (aligned with the child's visual system - VSMA) that displays visual information (augmented reality). Motion sensors capture the child's physical action (e.g., accelerometer). The system also captures physiological data - e.g., communicates wirelessly with a HR biosensor on the child's chest. Inputs to control virtual information (e.g., put an avatar into motion) are made through whole-body motion, e.g., the child may walk, run, jump, rotate, trot. Virtual information may also be controlled by interacting directly with sensor-based interfaces, e.g., manipulate a "turn button" or "bar" sensor; blow air into an "airflow sensor"; use a "glove sensor". "Albert" includes automatic feedback control mechanisms (software) similar to the mechanisms in "Cratus" (e.g., encouraging MVPA) (see Fig. 1).

In a cross-sectional pilot study ("Whole-body Interaction in Natural Environments Benefits Children's Cognitive Function Compared to Sedentary Interaction Indoors") presented in [13] - we compared the effects of whole-body interaction in a natural environment (stimulating the multiple sensory systems in the body) versus sedentary interaction in an indoor environment (stimulating mostly the visual and auditory senses) on cognitive function in a group of 10 typically developing children aged 7 to 8 (5 male and 5 female). Sustained attention and episodic memory (supporting long-term information storage - learning) were evaluated while each child interacted with a whole-body motion screen-based computer device ("Albert”), in a natural forested landscape, and a sedentary SBCD (desktop computer), based on hand-eye coordination skills, in a classroom setting (sitting at a desk) - video game play. Children's preferences and opinions regarding the interaction devices were also evaluated. Results indicate a trend for natural environment whole-body interaction to increase sustained attention in children over time, compared to indoor sedentary interaction. In turn, increases in sustained attention 
were associated with improvements in episodic memory during video game play. Children also preferred to interact with "Albert" in the NPE.

The latter results show that whole-body interaction in NPE is associated with benefits in children's cognitive function - learning through SBCDs seems to benefit from wholebody interaction in NPE. Interaction with sedentary SBCDs indoors may be failing to maximize children's learning in current educational systems.

The previous studies were approved by the Institutional Review Board at The University of Texas at Austin. All children agreed - by completing assent forms - to engage in the research studies; and that pictures and video data would be used targeting scientific purposes (scientific publications and presentations). The studies were judged to be of to minimal risk to children - not associated with psychological trauma, social or legal risks. We followed standard first aid practices. The main researcher was prepared to go through the following procedures in case of dehydration: direct the child to a cool and ventilated spot; apply cold compresses. In case of fatigue: reduce the intensity of the activity slowly; give small amounts of water to the child; move the child to a cool and ventilated spot.

\section{Conclusions}

We demonstrated that the current Child-Computer Interaction paradigm - interaction with SBCDs in artificially controlled environments - may be failing to optimize children's physical and mental health (including cognitive development and performance). From two initial studies, we observed that whole-body interaction in natural environments tends to benefit children's physical and mental health (including cognitive function). It is possible that modern children are still evolutionary constrained to favoring information processing in multimodal natural environments. We may have to rethink the current educational paradigm associated with child development through computing machines.

One of the main limitations of the first study - "Increasing Children's Physical Activity Levels Through Biosymtic Robotic Devices" - concerns its methodology - the evaluations were performed in a single experimental moment and were not repeated successively in order to validate the results. Hence, it is necessary to develop full-scale research studies (comprising large samples) in order to confirm the obtained results (including long-term spontaneous adherence to the device).

One of the main limitations of the second study - "Whole-body Interaction in Natural Environments Benefits Children's Cognitive Function Compared to Sedentary Interaction Indoors" - concerns its sample size (small sample). Future research is necessary to confirm the results. We also need to compare multimodal interaction indoors, versus natural environments, to understand if the latter represents a benefit for child cognition.

Acknowledgements. ST. Andrew's Episcopal School, UT Austin, FCT/UNL and Advanced Brain Monitoring. 


\section{References}

1. American Academy of Pediatrics. Policy Statement - Media Education. http:// pediatrics.aappublications.org/content/pediatrics/early/2010/09/27/peds.2010-1636.full.pdf

2. Armstrong, N., Welsman, J.: Development of aerobic fitness during childhood and adolescence. Pediatr. Exerc. Sci. 12, 128-149 (2000)

3. Baranowski, T., Abdelsamad, D., Baranowski, J., O'Connor, T.M., Thompson, D., Barnett, A., Cerin, E., Chen, T.-A.: Impact of an active video game on healthy children's physical activity. Pediatrics 129, 636-642 (2012)

4. Barsalou, L.W.: Grounded cognition. Annu. Rev. Psychol. 59, 617-645 (2008)

5. Bronstein, J.L.: Our current understand of mutualism. Q. Rev. Biol. 69(1), 31-51 (1994)

6. Calvert, G., Spence, C., Stein, B.E.: The Handbook of Multisensory Processing. The MIT Press, Cambridge (2004)

7. Chaddock, L., Erickson, K.I., Prakash, R.S., Kim, J.S., Voss, M.W., Vanpatter, M., Pontifex, M.B., Raine, L.B., Konkel, A., Hillman, C.H., Cohen, N.J., Kramer, A.F.: A neuroimaging investigation of the association between aerobic fitness, hippocampal volume and memory performance in preadolescent children. Brain Res. 1358, 172-183 (2010)

8. Chang, C.-H., Pan, W.W., Tseng, L.-Y., Stoffregen, T.A.: Postural activity and motion sickness during video game play in children and adults. Exp. Brain Res. 217, 299-309 (2012)

9. Chemero, A.: Radical Embodied Cognitive Science. The MIT Press, Cambridge (2009)

10. Chipman, G., Druin, A., Beer, D., Fails, J., Guha, M., Simms, S.: A case of study of Tangible Flags: a collaborative technology to enhance field trips. In: Proceedings of the 2006 Conference on Interaction Design and Children, IDC 2006, pp. 1-8. ACM, New York (2006)

11. Cleary, A.G., McKendrick, H., Sills, J.A.: Hand-arm vibration syndrome may be associated with prolonged use of vibrating computer games. Br. Med. J. 324, 301 (2002)

12. Cordes, C., Miller, E.: Fool's Gold: A Critical Look at Computers in Childhood. Alliance for Childhood (2002)

13. Ferraz, M., Resta, P., O'Neill, A.: Whole-body interaction in natural environments benefits children's cognitive function compared to sedentary interaction indoors (2016). Submitted for publication

14. Ferraz, M., Camara, A., O’Neill, A.: Increasing physical activity levels in children through biosymtic robotic devices. In: Proceedings of the 13th International Conference on Advances in Computer Entertainment Technology, ACE 2016. ACM, New York (2016)

15. Fitzpatrick, G., Fleck R., Harris, E., Muller, H., O`Malley, C., Price, S., Rogers, S., Smith, H., Randell, C., Stanton, D., Thompson, M., Weal, M.: Ambient wood: designing new forms of digital augmentation for learning outdoors. In: Proceedings of the 2004 Conference on Interaction Design and Children: Building a Community, IDC 2004, pp. 3-10 (2004)

16. Gibson, J.J.: The Ecological Approach to Visual Perception. Lawrence Erlbaum Associates Inc., New York and London (1979)

17. Goldin-Meadow, S., Nusbaum, H., Kelly, S.D., Wagner, S.: Explaining math: gesturing lightens the load. Psychol. Sci. 12, 516-522 (2001)

18. Granic, I., Lobel, A., Engels, R.: The benefits of playing video games. Am. Psychol. 69(1), 66-78 (2014)

19. Green, C.S., Bavelier, D.: The development of attention skills in action video game players. Neuropsychologia 47(8-9), 1780-1789 (2009)

20. Hamlen, K.: Children's choices and strategies in video games. Comput. Hum. Behav. 27, 532$539(2011)$ 
21. Hillman, C.H., Kamijo, K., Scudder, M.: A review of chronic and acute physical activity participation on neuroelectric measures of brain health and cognition during childhood. Prev. Med. 52, S21-S28 (2011)

22. Kuo, F.E., Faber, A.: A Potential natural treatment for attention-deficit/hyperactivity disorder: evidence from a national study. Am. J. Pub. Health 94, 1580-1586 (2004)

23. LeBlanc, A.G., Chaput, J.-P., McFarlane, A., Colley, R.C., Thivel, D., Biddle, J.H., Maddison, R., Leatherdale, S.T., Tremblay, M.S.: Active video games and health indicators in children and youth: a systematic review. PLoS ONE 8(6), e65351 (2013). doi:10.1371/journal.pone. 0065351

24. Lewkowicz, D., Roder, B.: Development of multisensory processing and the role of early experience. In: Spence, C., Stein, B.E. (eds.) The Handbook of Multisensory Processing, pp. 607-626. The MIT Press, Cambridge (2012)

25. Lieberman, E.D.: The Story Of The Human Body. Evolution, Health and Disease. Penguin Books, London (2013)

26. Liu, T.-C., Lin, Y.-C., Tsai, M.-J., Pass, F.: Split-attention and redundancy effects on mobile learning in physical environments. Comput. Educ. 58(1), 172-180 (2012)

27. Lou, D.: Sedentary behaviors and youth: current trends and the impact on health, pp. 1-12. Active Living Research, San Diego (2014)

28. Lumley, M.A., Relamed, B.G., Abeles, L.A.: Predicting children's presurgical anxiety and subsequent behavior changes. J. Pediatr. Psicol. 18, 481-497 (1993)

29. Magerkurth, C., Cheok, A.D., Mandryk, R.L., Nilsen, T.: Pervasive games: bringing computer entertainment back to the real world. Comput. Entertainment (CIE) Theor. Pract. Comput. Appl. Entertainment 3(3), 4 (2005)

30. Malinverni, L., López, S.B., Parés, N.: Impact of embodied interaction on learning processes: design and analysis of an educational application based on physical activity. In: Proceedings of the 11th International Conference on Interaction Design and Children, IDC 2012, pp. 6069. ACM, New York (2012)

31. Montagu, A.: Touching: The Human Significance of the Skin, 2nd edn. Harper and Row, New York (1972)

32. Mueller, F., Gibbs, M.R., Vetere, F.: Taxonomy of exertion games. In: Proceedings of the 20th Australasian Conference on Computer-Human Interaction: Designing for Habitus and Habitat, OZCHI 2008, pp. 263-266. ACM, New York (2008)

33. Noble, J.: Programming Interactivity. A Designer's Guide to Processing, Arduino, and OpenFrameworks. O'Reilly, California (2009)

34. Piek, J.P., Dawson, L., Smith, L.M., Gasson, N.: The role of early fine and gross motor development on later motor and cognitive ability. Hum. Mov. Sci. 27(5), 668-681 (2008)

35. Rajakumar, K.: Vitamin D, cod-liver oil, sunlight, and rickets: a historical perspective. Pediatrics 112(2), 132-135 (2003)

36. Rideout, V.J., Foehr, U.G., Roberts, D.F.: Generation $\mathrm{M}^{2}$ : Media in the Lives of 8 to 18 -YearOlds. Kaiser Family Foundation, Menlo Park (2010)

37. Rose, K.A., Morgan, I.G., Ip, J., Kifley, A., Huynh, S., Smith, W., Mitchell, P.: Outdoor activity reduces the prevalence of myopia in Children. Ophthalmology 115(8), 1279-1285 (2008)

38. Rowland, T., Bar-Or, O.: Pediatric Exercise Medicine. Physiologic Principles to Health Care Application. Habitual activity and Energy Expenditure in the Healthy Child. Human Kinetics Pub Inc, Champaign (2004)

39. Small, G., Vorgan, G.: IBrain: Surviving the Technological Alteration of the Modern Mind. Harper Collins Publishers, New York (2008) 
40. Stratton, G.: Review articles physical activity levels in middle and high school physical education: a review. Pediatr. Exerc. Sci. 17, 3 (2005)

41. Sweller, J.: Instructional Design in Technical Areas. ACER Press, Camberwell (1999)

42. Thelen, E., Smith, L.B.: A Dynamical Systems Approach to the Development of Cognition and Action. The MIT Press, Cambridge (1994)

43. UCLA Center to Eliminate Health Disparities and Samuels and Associates: Failing Fitness: Physical Activity and Physical Education in Schools. Policy Brief. The California Endowment, Los Angeles (2007)

44. Verstraete, S.J.M., Cardon, G.M., De Clercq, D.L.R., De Bourdeaudhuil, M.M.: Increasing children's physical activity levels during recess in elementary schools: the effects of providing game equipment. Eur. J. Public Health 16(4), 415-419 (2006)

45. World Health Organization. Obesity and Overweight. http://www.who.int/ dietphysicalactivity/childhood/en/

46. World Health Organization: Physical Activity and Young People (2013). http://www.who.int/ dietphysicalactivity/factsheet_young_people/en/

Open Access This chapter is licensed under the terms of the Creative Commons Attribution 4.0 International License (http://creativecommons.org/licenses/by/4.0/), which permits use, sharing, adaptation, distribution and reproduction in any medium or format, as long as you give appropriate credit to the original author(s) and the source, provide a link to the Creative Commons license and indicate if changes were made.

The images or other third party material in this chapter are included in the chapter's Creative Commons license, unless indicated otherwise in a credit line to the material. If material is not included in the chapter's Creative Commons license and your intended use is not permitted by statutory regulation or exceeds the permitted use, you will need to obtain permission directly from the copyright holder.

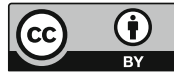

